\title{
Open Education
}

SPARC

\section{Source}

https://sparcopen.org/open-education/SPARC

Open Education encompasses resources, tools and practices that are free of legal,

financial and technical barriers and can be fully used, shared and adapted in the digital environment. Open Education maximizes the power of the Internet to make education more affordable, accessible and effective. 